\title{
Ictus isquémico y disección bilateral de la arteria carótida interna: una manifestación rara del síndrome de Eagle. Reporte de un caso

\author{
Ischemic stroke and bilateral dissection of the internal carotid artery: \\ A rare manifestation of the Eagle syndrome. A case report
}

\author{
Claudio Carnevale $\mathbf{S}^{\mathbf{1}}$, Carmen Jiménez $\mathbf{M}^{2}$, Meritxell Tomás $\mathrm{F}^{1}$, Diego Arancibia $\mathrm{T}^{1}$, Guillermo Til $\mathbf{P}^{1}$, \\ Pedro Sarría E1, Manuel Tomás B1.
}

\begin{abstract}
RESUMEN
Un síndrome caracterizado por dolor cervical y un apófisis estiloides alargado fue descrito por primera vez por Watt Eagle en 1937. Aunque el síndrome de Eagle en su variante vascular es raro y no es reconocido como causa clara de disección carotídea, en los últimos años ha sido reportado un incremento del número de casos de disección carotídea causada por una apófisis estiloides alargada.

Paciente de 56 años que acudió al servicio de urgencias por paresia facial izquierda aguda y habla confusa. Presentaba dolor cervical de dos días de evolución, relacionado con un ataque de tos. Se activó el código ictus y la tomografía computarizada (TC) mostró isquemia del lóbulo temporal derecho y disección bilateral de la arteria carótida interna. La angio-TC de los troncos supraaórticos con reconstrucción tridimensional, identificó una apófisis estiloides alargado en ambos lados. El paciente fue sometido a una angioplastía con colocación de dos stents. Debido al alto riesgo de padecer nuevo ictus, se decidió realizar tratamiento quirúrgico. Para el lado derecho se realizó un abordaje transoral y en el izquierdo un abordaje abierto.

La apófisis estiloides alargado es una causa importante de disección carotídea y de las complicaciones cerebrovasculares relacionadas.
\end{abstract}

Palabras clave: Eagle, dolor cervical, disección de la arteria carótida, ictus; apófisis estiloides.

\begin{abstract}
A syndrome characterized by cervical pain and an abnormally elongated styloid process was first described by Watt Eagle in 1937. Even though vascular Eagle syndrome
\end{abstract}

\footnotetext{
1 Servicio de Otorrinolaringología y Cirugía de Cabeza y Cuello, Hospital Universitario Son Espases, Palma de Mallorca, España.

2 Servicio de Neurología, Hospital Universitario Son Espases, Palma de Mallorca, España.
}

Los autores declaran no tener conflictos de interés.

Recibido el 8 de marzo, 2019. Aceptado el 16 de abril, 2019. 
is uncommon and is not well recognized as a cause for carotid artery dissection, in the last few years there have been an increasing number of case reports of carotid artery dissection caused by an elongated styloid process.

A 56 years old man presented to the emergency department with acute left facial weakness and slurred speech. He complained of two days neck pain, related to a coughing fit. The code stroke protocol was activated and imaging showed a right temporal bone stroke and a bilateral internal carotid artery dissection. A scan angiography of the supra-aortic vessels with $3 D$ reconstruction was performed showing a bilateral abnormally elongated styloid process. The patient underwent angioplasty with two stent placements. Due to the high risk of recurrent strokes, the patient was evaluated in the otolaryngology department for styloid process resection. Finally a transoral approach for the right side and an open approach for the left side were performed.

We have to consider an elongated styloid process as an important cause of carotid artery dissection and subsequent cerebrovascular complications

Key words: Eagle, cervical pain, carotid artery dissection, stroke, styloid process.

\section{INTRODUCCIÓN}

El síndrome de Eagle debe su nombre al otorrinolaringólogo Watt Eagle que por primera vez describió la asociación entre un alargamiento anormal de la apófisis estiloides 0 del ligamento estilo-hioideo calcificado y los síntomas relacionados con la compresión de las estructuras cercanas. La apófisis estiloides es una prominencia ósea localizada en la porción inferior del hueso temporal que se extiende en dirección antero-medial y caudalmente cerca de las arterias carótidas, de la vena yugular interna y de los nervios craneales V, VII, IX, X y XII${ }^{3}$. Los síntomas son clásicamente causados por el pinzamiento de estructuras nerviosas o vasculares por parte de una apófisis estiloides de longitud aumentada. Esta última se considera de longitud aumentada si mide más de $3 \mathrm{~cm}$ y en caso de coexistir síntomas es posible hacer un diagnóstico de síndrome de Eagle. No toda apófisis estiloides alargada causa síntomas. La incidencia de una apófisis estiloides alargada varía entre $4 \%$ y $28 \%$ en la población general y es vista sobre todo en mujeres ( 3 veces más frecuente que en hombres), con un pico de incidencia entre los 60 y 79 años, pero sólo una minoría de los pacientes son sintomáticos². El síndrome se divide en dos categorías según la etiopatogenia de la sintomatología: el síndrome clásico y el síndrome estilo-carotídeo (Tabla 1).

El primero se caracteriza por la compresión nerviosa y la estimulación de los nervios V, VII, IX, $X$ y XII, causando odinofagia, disfagia, sensación de cuerpo extraño, y dolor cervical que pueden aumentar de intensidad con los movimientos de la cabeza.

Tabla 1. Síntomas del síndrome de Eagle según la variante de presentación

\begin{tabular}{|lll|}
\hline & Variante clásica & Variante vascular (estilo-carotídeo) \\
\hline Sexo & F>M & F>M \\
Edad & $50-60$ años & $50-60$ años \\
Odinofagia/disfagia & Común & No observado \\
Dolor & Parietal hasta la región pectoral & Región periorbitaria \\
Estridor & Raro & No observado \\
Vértigo & No observado & Común \\
Síncope & No observado & Común \\
Flexión/extensión & Los síntomas aumentan & Los síntomas aumentan \\
Rotación contralateral de la cabeza & Mínimo efecto sobre los síntomas & Los síntomas aumentan \\
Palpación de la fosa amigdalina & Los síntomas aumentan & Mínimo efecto sobre los síntomas \\
\hline
\end{tabular}


El segundo, que es la variante menos frecuente, es debido a la compresión vascular que causa la irritación de las fibras del plexo simpático pericarotídeo. Se puede caracterizar por los mismos síntomas que el síndrome clásico, pero el dolor presenta una distribución característica a lo largo de la arteria comprometida. Si está comprometida la arteria carótida interna el dolor se irradia hacia las regiones supraorbitaria y parietal, mientras si está afectada la arteria carótida externa el dolor se irradia hacia la región infraorbitaria. Esta condición se puede complicar con la disección de la arteria carótida y tromboembolismo, resultando en eventos cerebrovasculares con alteraciones de la visión, disfunción motora, afasia, síncope y dolor cervical severo que se irradia al oíd0 ${ }^{4-6}$. Describimos un caso de un paciente que presentó un ictus isquémico derecho y fue diagnosticado de disección bilateral de la arteria carótida interna relacionada con un síndrome de Eagle bilateral.

\section{CASO CLÍNICO}

Un paciente de 56 años con antecedente de hipertensión arterial acudió al servicio de urgencias con paresia facial izquierda aguda y habla confusa. Él refería dolor cervical de dos días de evolución relacionado con un ataque de tos. A la exploración física el paciente presentaba además de la paresia facial izquierda, también debilidad y entumecimiento del miembro superior ipsilateral. Ante la sospecha de un ictus isquémico cerebral se realizaron una tomografía computarizada (TC) con estudio de perfusión y una angio-TC de los troncos supraaórticos con reconstrucción 3D. La TC craneal mostró una pérdida focal de diferenciación córtico-subcortical en el córtex insular derecho y en el polo anterior del lóbulo temporal derecho. Se empezó de forma inmediata tratamiento fibrinolítico endovenoso, que se interrumpió después de confirmar en la angio-TC la existencia de un ictus agudo debido a un embolismo secundario a la disección de la arteria carótida interna derecha. De hecho, en la angio-TC se observaron en el lado derecho la oclusión de la arteria cerebral media con una oclusión posbulbar de la arteria carótida interna ipsilateral relacionada con disección y, en el lado izquierdo, un pseudoaneurisma de la arteria carótida interna posbulbar y una disección a nivel de la primera vértebra cervical. Por este motivo se procedió a la trombectomía mecánica endovascular. Durante el ingreso hospitalario se realizó nuevamente una angioTC de los troncos supraaórticos con reconstrucción 3D que mostró una apófisis estiloides alargada bilateral, de $3,4 \mathrm{~cm}$ en el lado derecho y $3,8 \mathrm{~cm}$ en el lado izquierdo, en contacto con la arteria carótida interna, sugiriendo un síndrome estilo-carotídeo (Figura 1).

Durante el seguimiento se realizaron una resonancia magnética (RM) cerebral y una angio-RM que mostraron signos de isquemia crónica de la ínsula derecha en el territorio de la arteria cerebral media con

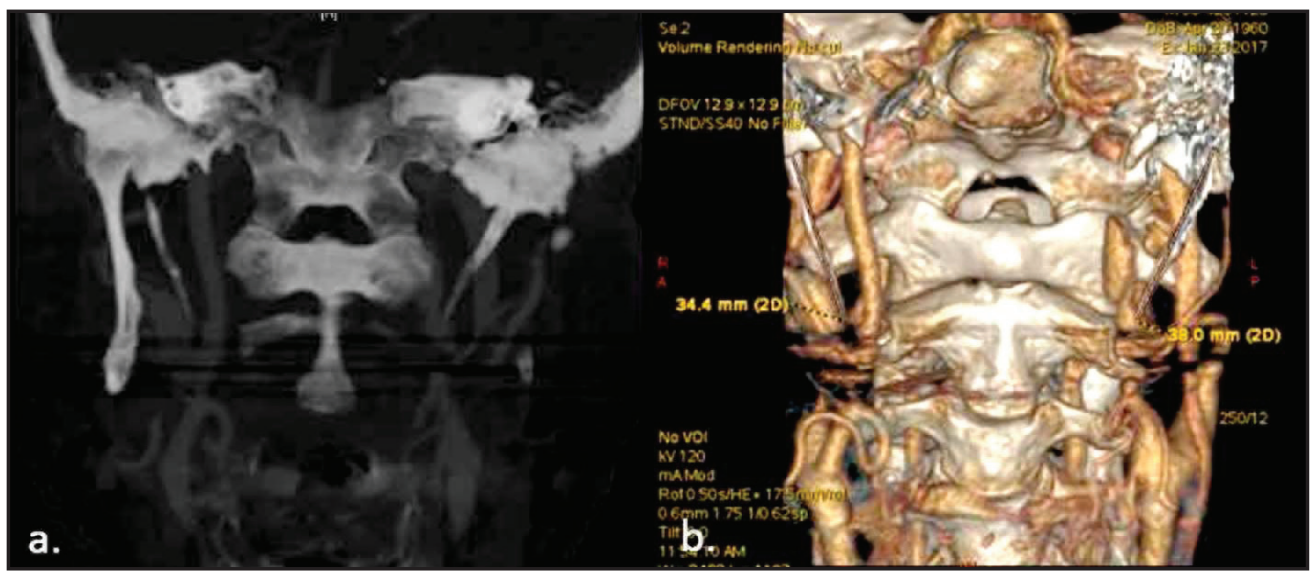

Figura 1. (a) Angio-TC de los troncos supraaórticos. (b) TC con reconstrucción 3D. Se observa bilateralmente la relación entre la apófisis estiloides de longitud aumentada y la arteria carótida interna. 
disección persistente de ambas carótidas internas y una estenosis significativa focal de la arteria carótida interna izquierda asociada con pseudoaneurisma proximal. Por eso el paciente fue sometido a una angioplastía endovascular con el posicionamiento de dos stents.

Debido al alto riesgo de ictus recurrentes, el paciente fue evaluado en el servicio de otorrinolaringología y se decidió intervención quirúrgica. Se realizó un abordaje transoral en el lado derecho. El lado izquierdo fue intervenido a través de un abordaje abierto por cervicotomía dos meses después, con el fin de conseguir un mayor control de los grandes vasos (en este lado se había observado un "kinking" carotídeo). Después de realizar una cervicotomía izquierda, una disección cuidadosa permitió identificar la vena yugular interna, las carótidas interna y externa, y los nervios craneales XI y XII. Después de ligar la vena yugular interna, el vientre posterior del músculo digástrico fue cortado para acceder al espacio parafaríngeo y el apófisis estiloides fue identificado y resecado (Figura 2). El paciente actualmente se mantiene asintomático con resolución completa de los síntomas neurológicos después de un seguimiento de 1,5 años.

\section{DISCUSIÓN}

A pesar de que entre el $4 \%$ y el $28 \%$ de la población general presente una apófisis estiloides mayor de $3 \mathrm{~cm}$, sólo el 4\% de ellos presentan síntomas, lo que corresponde al $0,16 \%$ de la población general ${ }^{4}$. En la mayoría de los casos se observa una apófisis alargada en ambos lados, pero los síntomas suelen ser unilaterales. Aunque el síndrome de Eagle vascular es poco frecuente y no es reconocido como una causa clara de disección carotídea, en los últimos años ha habido un incremento de los casos descritos de disección carotídea relacionados con una apófisis estiloides de longitud aumentada. En diferentes estudios, la disección de la arteria carótida se ha relacionado con la longitud aumentada de la apófisis estiloides y con una distancia disminuida entre la apófisis y la arteria carótida interna ${ }^{5}$.

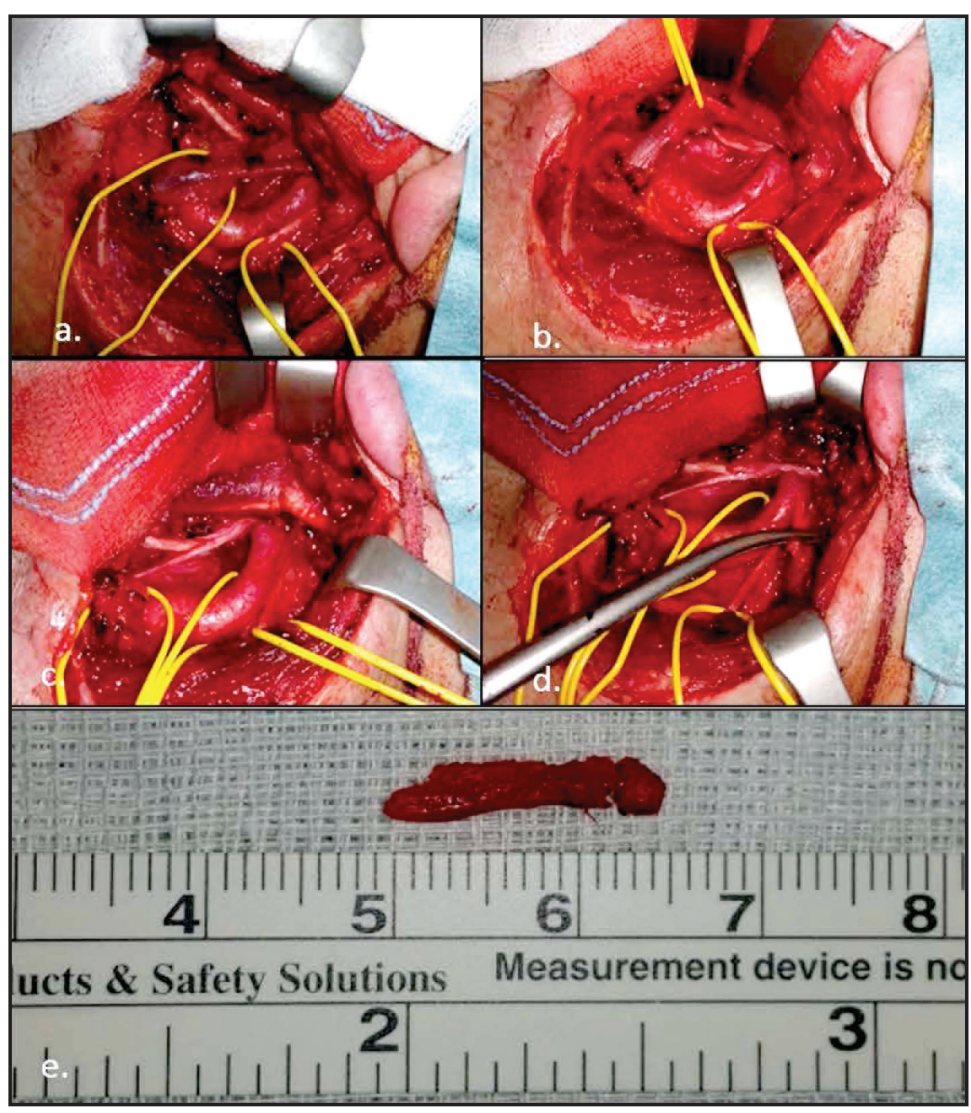

Figura 2. (a) Identificación de la vena yugular interna, de la arteria carótida, de los nervios hipogloso y espinal. (b) Disección de la vena yugular interna. (c) Ligadura de la vena yugular interna. El vientre posterior del músculo digástrico ha sido cortado. (d) El apófisis estiloides ha sido cortada. (e) Fragmento resecado del apófisis estiloides. 
El diagnóstico de disección carotídea causado por el síndrome de Eagle es difícil. La angio-TC con reconstrucción 3D es el gold standard, pero no es realizado de rutina en pacientes con disección carotídea. A pesar de que la estrategia terapéutica para la disección carotídea causada por el síndrome de Eagle no es bien establecida, el tratamiento de primera línea para un ictus isquémico asociado con un síndrome de Eagle vascular tendría que ser la terapia anticoagulante ${ }^{6}$. Cuando los pacientes presentan ictus recurrentes a pesar del tratamiento médico 0 refieren dolor, la resección quirúrgica de la apófisis estiloides tendría que ser considerada como en nuestro caso. El empleo de stents carotídeos podría ser otra opción, especialmente en pacientes que han sufrido una oclusión súbita de la arteria carótida. En cualquier caso, no se ha demostrado su eficacia y han sido descritos trombosis y tromboembolismos relacionados con su colocación. La resección quirúrgica de la apófisis o proceso estiloides tiene el objetivo de descomprimir las arterias carótidas y prevenir nuevos eventos cerebrovasculares. El procedimiento puede ser realizado por vía transoral 0 cervical7. La

\section{BIBLIOGRAFÍA}

1. EAGLE WW. Elongated styloid process: Symptoms and treatment. AMA Arch Otolaryngol 1958; 67: 172-6.

2. Keur JJ, Campbell JP, McCarthy Jf, Ralph WJ. The clinical significance of the elongated styloid process. Oral Surg Oral Med Oral Pathol 1986; 61: 399-404.

3. Farhat HI, Elhammady MS, Ziayee H, Aziz-Sultan MA, Heros RC. Eagle syndrome as a cause of transient ischemic attacks. J Neurosurg 2009; 110: 90-3.

4. EAGLE WW. Symptomatic elongated styloid process; report of two cases of styloid processcarotid artery syndrome with operation. Arch Otolaryngol 1949; 49: 490-503.

5. Raser JM, Mullen Mt, Kasner Se, Cucchiara BL, Messé SR. Cervical carotid artery dissection is associated with styloid process length. Neurology 2011; 77: 2061-6. elección depende de la anatomía y de la preferencia del cirujano, con tasas de éxito parecidas. Cada uno de estos abordajes presenta ventajas y desventajas. El abordaje abierto por cervicotomía ofrece una mejor exposición quirúrgica con un riesgo inferior de infección y de lesión de las estructuras vasculares y nerviosas del cuello. Su mayor desventaja es la cicatriz y la posibilidad de un resultado estético pobre. En nuestro caso, la realización de un abordaje transoral fue posible sólo en el lado derecho, a través de una disección cuidadosa bajo visión microscópica.

\section{CONCLUSIÓN}

Hasta ahora sólo han sido descritos tres casos de disección bilateral de la arteria carótida interna causada por una apófisis estiloides de longitud aumentada ${ }^{1,8-10}$. Este caso pone en evidencia la importancia de considerar la apófisis estiloides de longitud aumentada como causa en el diagnóstico diferencial de disección carotídea y de sus complicaciones cerebrovasculares.

6. Todo T, Alexander M, Stokol C, Lyden P, Braunstein G, Gewertz B. Eagle syndrome revisited: cerebrovascular complications. Ann Vasc Surg 2012; 26: 729e1-729e5.

7. Han MK, KIm DW, Yang JY. Non surgical treatment of Eagle's syndrome-a case report. Korean $J$ Pain 2013; 26: 169-72.

8. Badhey A, Jategaonkar A, Anglin Kovacs AJ, Kadakia S, De Deyn PP, Ducic Y, Schantz S, Shin E. Eagle syndrome: a comprehensive review. Clin Neurol Neurosurg 2017; 159: 34-8.

9. Naito $Y$, YamazaKI K. Perioperative management of Eagle syndrome complicated by carotid artery dissection. J Anesth 2014; 28: 291-3.

10. Jelodar S, Ghadirian H, Ketabchi M, Ahmadi Karvigh S, Alimohamadi M. Bilateral Ischemic Stroke Due to Carotid Artery Compression by Abnormally Elongated Styloid Process at Both Sides: A Case Report. J Stroke Cerebrovasc Dis 2018; 27: 89-91.

Correspondencia: Claudio Carnevale Serao

Secretaria del Servicio de Otorrinolaringología y Cirugía de Cabeza y Cuello, Hospital Universitario Son Espases, Carretera de Valldemossa 79, 07210, Palma de Mallorca, España.

E mail: claudio.carnevale.orl@gmail.com 